\title{
PANEL ON XML AND SECURITY
}

\author{
Sylvia Osborn \\ The University of Western Ontario, London, Canada \\ sylvia@csd.uwo.ca
}

Bhavani Thuraisingham

Mitre Corporation, USA

thura@mitre.org

Pierangela Samarati

Dip. di Tecnologie dell'Informazione - Università di Milano, 20163 Crema, Italy

samarati@dti.unimi.it

\section{INTRODUCTION BY SYLVIA OSBORN}

The panelists were asked to address the question: "XML security: are all the problems solved?" As we can see from the following sections, a large number of issues are being addressed by the www community as well as by the database security community related to XML and security. Both contributers indicate that there is a great deal of interesting research still to be done in this area.

\section{POSITION BY B. THURAISINGHAM}

Recently there has been increasing interest in developing access control models for XML documents. Since XML is becoming the standard document representation for the web, and XML extensions are being proposed for all types of documents including multimedia documents, it is critical that these documents are secure.

There are various aspects to securing XML documents. These include encryption, access control, and authentication. In providing access control for XML documents, one of the major challenges is the level of granularity. Should entire XML documents be protected or should access be granted depending on the content of the document, or to portions of the document? For example if John cannot have access to say information represented by a node in an XML document, then should John be denied access to all information emanating from 
that node? How can access be granted based on content of the document? What is the access control policy for XML documents? How should XML documents be updated? Is there an update policy for XML documents? How can inference threats be handled for XML documents? How can role-based access control policies be implemented?

Various standards groups are now working on securing XML documents. One question that arises often is what is the difference between securing XML documents and securing object models? In fact we can learn a lot from our experiences in developing access control for object database systems. We need to reuse the known techniques and models as much as possible if they make sense for XML documents. However XML also has its own features including XML and document type definitions. Therefore we need to ensure that appropriate security mechanisms are developed for XML documents.

Closely related to XML security is security for RDF (Resource Description Framework) and the Semantic Web. The World Wide Web consortium is actively involved in coming up with RDF specifications and providing the directions for the semantic web. XML security is just the first step toward securing the semantic web. We need to examine security for RDF, agents and many other technologies before we can develop security policies for the semantic web. It is critical that we examine security for the semantic web while various specifications are being developed.

\section{POSITION BY PIERANGELA SAMARATI}

Several works have recently addressed the topics of XML and security. With reference to authorization-based approaches, the relationships between the two topics can be distinguished as:

- protecting XML documents;

- protecting XML-based services;

- $\mathrm{XML}$ as a security specification language.

\section{Protecting XML documents}

Recent years have witnessed a tremendous growth in Web publishing and Web-based applications. At the early stage, Web publishing was associated with the release of information to be made publicly available. The subsequent increased use of the Web as a data communication medium introduced the need for selective publishing where documents and portions of them needed to be made accessible only to specific requestors. However, the variety of the format of documents to be published and the lack of a structure for them limited the specification of access restrictions: documents were simply viewed as 
files and their protection enforced by the operating system. For instance, the Apache server (www.apache.org) allows the specifications of access control lists via a configuration file containing the list of users, hosts (IP addresses) or host/user pairs, that must be allowed or forbidden connection to the server. The consideration of both positive and negative authorizations, together with a user-specified priority order for resolving conflicts among them and establishing a default policy ("allow, deny" vs "deny, allow"), introduce some flexibility in the specification of protection requirements [10]. However, the coarse granularity at which authorizations are supported does not addresses real protection needs, as a single document may indeed contain data with different protection requirements and enforcing them would require splitting the document in different files, possibly organized in different directories, each subject to its own security specifications. While this solution can reach the intended result, it is quite awkward and difficult to administer in practice. An attempt to provide a finer grained access control was proposed in [9] allowing the specification of authorizations with reference to specific portions of HTML documents (delimited by properly defined tags). However, conventional HTML tagging is aimed at defining page rendering and is seldom, if ever, related to information granulation. In fact HTML provides no clean separation between the structure and the layout of the document. Moreover, site designers often prepare HTML pages according to the needs of a particular browser. Therefore HTML markup has generally little to do with data semantics. The introduction and wide diffusion of XML (eXtensible Markup Language), maintaining formatting and rendering capabilities while providing semantics-aware markup techniques, represented the opportunity to overcome the limitation of free formatting in expressing access control restrictions. The attention of access control research therefore shifted to specification and enforcement of authorizations for XML documents.

XML provides several advantages. First, it allows reference to specific elements and attributes within the documents, thus permitting the specification and enforcement of different access restrictions within each document. Such capability is further made more advantageous by the ability of exploiting languages (such as XPath) to identify elements and attributes within a document in an expressive way. By exploiting such languages, not only authorizations can be referred to specific elements/attributes within a document, but the elements/attributes to which an authorization refers can be specified based on properties they satisfy (content-dependent authorizations). For instance, an authorization could specify that all employees could access the details (results and funding) of internal research on the web library, where whether research is considered 'internal' is specified as a value of some element in the documents. A further advantage of exploiting XML for access control is given by the hierarchical relationship [7] between document instances and the related DTD/schema, that allows the support of authorizations for single documents as well as sets 
of them. Several proposals have therefore been studied which joined current trends in providing flexibility in authorizations [10] with the fine-granularity and expressiveness advantages of XML and related query languages.

The definition of an access control model for XML documents pointed out several issues to be addressed, including:

- The identification of different semantics for authorizations related to different propagation options, such as in the case of element-level authorizations which may need to be supported with a recursive or local semantics (depending on whether authorizations on an element propagate on its sub-elements).

- The identification of different resolution policies for solving conflicts between authorizations. Among the policies to be supported, the one based on the most specific relationship among authorization objects (e.g., a document is more specific than its schema, and an attribute is more specific than the element in which it is defined) like in traditional hierarchical systems [7]. Since no single solution can fit all the needs, a way of subverting the predefined policy should be supported [2] together with alternative solutions $[1,6,7]$.

- The identification of processing techniques for enforcing authorizations and determining the view to be returned to requestors. Among the issues to be addresses in this respect is how to deal with cases where an element should not be returned while part of its content should. In fact, authorization enforcement could lead to the result that a document's element (e.g., an article) should not be released to a requestor but one of its subelement (e.g., the article's abstract) should be (e.g., because of a more specific positive authorization overriding the negative authorization on the element). What should the system do? At least three different approaches could be identified: 1) return the sub-element but completely hide the element; 2 ) return the sub-element and the tag for the element, to preserve the document's structure; 3 ) hide the element together with its whole content (including the sub-element which could instead be released). The first solution, while strictly enforcing the requirements, has the drawback of loosing the document's structure and therefore the semantics of the data returned. Based on this argument, the second solution was adopted in [2] returning the start and end tag of elements with undefined or negative authorizations that have a descendant with a positive authorization. The third approach was instead proposed in the context of SOAP call filtering [4] (cf. next section).

- The identification of where authorizations are stored. Two different approaches could be taken in this respect. The first solution consists in 
attaching authorizations directly to the elements on which they are specified. This is, for instance, what happens today where specific pairs userpassword are associated with portions of HTML web-pages. It is also the solution adopted for the specification of fine-grained encryption [5]. The main drawback of such an approach is that it does not obey the principle of separation between data (and data model) and security specification [10]. An alternative approach attaches to each document/schema an authorization sheet [2].

- The definition of the authorization's format and syntax. Exploiting XML's own capability, several proposals have adopted recursive approaches, where authorizations are expressed in $X M L$. These approaches have started the line of efforts on the definition of an XML-based authorization language.

Several points still remains to be fully investigated. Among them:

- Balancing expressiveness and performance. Enforcing access control requires the evaluation of the path expressions in the authorizations and the computation of the view to be returned to requestors based on authorizations applicable to the different elements/attributes. Performance issues become particularly critical, since XPath is a rich programming language that permits the definition of search expressions requiring exponential time for their evaluation. However, several works identify restrictions on XPath that reduce its complexity characteristics, keeping a level of expressibility adequate for most situations [2]. Another issue to be considered with reference to performance is how to actually compute the document view. For instance, [2] uses an approach based on the use of the Document Object Model (DOM), computing and uploading the DOM for the document for authorization enforcement.

- Extending actions. Although modification actions (write and update) have been considered by recent work for XML document protection [2], no consensus has emerged up to now in the research community on a model for XML updates. As research progresses there, access controls for update operations can be refined accordingly.

- Query-based access control. All approaches consider access control on a document basis (assuming each request refers to a specific document). In several applications, requests may be submitted as queries spanning several documents or portions of them. Access control should therefore be extended to the consideration of such requests. 


\section{Securing XML-based e-services}

XML-based approaches have been proposed for lightweight network protocols and distributed applications, such as XML-RPC (www.xmlrpc.com) and SOAP (www.w3.org/2000/xp). For instance, SOAP defines a mechanism to pass commands and parameters between HTTP clients and servers and uses $\mathrm{XML}$ for data encoding and is therefore independent from the operating system, programming language, or object model used on either the server or the client side. Leveraging the XML encoding used by SOAP for both service invocations and responses, Damiani et al. [4] proposed an approach for protecting SOAP-based e-services. The approach extended and refined the author's proposal for protecting XML documents. Every SOAP invocation and its response are intercepted by the access control module that evaluates them against the specified restrictions to service accessibility. Depending on the restrictions to be enforced the request may: be rejected; be allowed as is; be filtered or executed in a modified form, where filtering of a request may involve elimination of some of its parameters that the current invoker is not allowed to specify. Service requestors are characterized by custom headers included in SOAP calls which accommodate for specifying the subjects identity, their roles, and possible digital certificates they can present. The specification of security restrictions directly on invocations and their responses provided a way of avoiding firewall dependence for the enforcement of security restrictions and of supporting finegrained security constraints.

\section{XML as a security specification language}

Recent research in access control has been targeted to enriching the expressiveness and the flexibility of the authorization specification language. Most effective proposals are based on the use of logic languages (e.g., [7]). Logicbased proposals provide the needed flexibility and expressiveness; however they are not always easily accepted by end users. The wide accepted use of XML, its flexibility, expressiveness, and interoperability brought to efforts investigating the use of XML as a security specification language. The use of XML as an authorization specification language was already noticed within proposals for protecting XML documents, which recursively represented authorizations as XML documents $[2,8]$. As another example, ContenGuard is developing an eXtensible Rights Markup Language (XrML) 2.0 (www.xrml.org) for finegrained rights control on document-like content and on Web-based services.

OASIS (Organization for the Advancement of Structured Information Standards), a nonprofit international consortium that creates interoperable industry specifications based on public standards, has recently started a standardization effort on the use of XML-based languages to express and interchange access control policies. The effort is carried out by the OASIS eXtensible Access 
Control Markup Language (XACML) technical committee whose goal is to define a standard core schema and corresponding namespace for the expression of authorization policies in XML against objects that can be themselves identified in XML. Exploiting the flexibility of XML, the committee is studying the definition of a schema that can represent existing authorization specification approaches and that can express different protection requirements. Moreover, the semi-structured feature of XML provides a powerful means for specifying authorizations whose validity depends on properties that requestors can satisfy (like enjoying a role, or belonging to a group).

\section{Acknowledgments}

Pierangela Samarati would like to thank Ernesto Damiani, Sabrina De Capitani di Vimercati, and Stefano Paraboschi: the observations reported here result from discussions and joint work with them.

\section{References}

[1] E. Bertino, S. Castano, and E. Ferrari Securing XML documents with author-X. IEEE Internet Computing, 5(3):21-31, May/June 2001.

[2] E. Damiani, S. De Capitani di Vimercati, S. Paraboschi, and P. Samarati. A fine-grained access control system for $\mathrm{xml}$ documents. ACM Transactions on Information and System Security, 2000. (to appear).

[3] E. Damiani, S. De Capitani di Vimercati, S. Paraboschi, and P. Samarati Securing XML Documents. Proc. of EDBT 2000, Volume 1777 of Lecture Notes in Computer Science, Konstanz, Germany, March 2000.

[4] E. Damiani, S. De Capitani di Vimercati, S. Paraboschi, and P. Samarati. Securing SOAP e-services. International Journal of Information Security (IJIS), 2002. (to appear).

[5] D. Eastlake, J. Reagle, and D. Solo. XML-Signature Syntax and Processing, March 2001. ftp://ftp.rfc-editor.org/in-notes/rfc3075.txt.

[6] A. Gabillon and E. Bruno. Regulating access to XML documents. In Proceedings of the Fifteenth Annual IFIP WG 11.3 Conference on Database Security, Niagara on the Lake, Ontario, Canada, July 2001.

[7] S. Jajodia, P. Samarati, M.L. Sapino, and V.S. Subrahmanian. Flexible support for multiple access control policies. ACM Transactions on Database Systems, 26(2):214-260, June 2001.

[8] M. Kudoh and S. Hada. Xml document security based on provisional authorizations. In ACM Computer and Communications Security Conference, (ACM CCS'2000), Athens, Greece, November 2000.

[9] P. Samarati, E. Bertino, and S. Jajodia. An authorization model for a distributed hypertext system. IEEE Transactions on Knowledge and Data Engineering, 8(4):555-562, August 1996.

[10] P. Samarati and S. De Capitani di Vimercati. Access control: Policies, models, and mechanisms. In R. Focardi and R. Gorrieri, editors, Foundations of Security Analysis and Design, LNCS 2171. Springer-Verlag, 2001. 